\title{
Preliminary thermal-hydraulic analysis of the EU-DEMO Helium-Cooled Pebble Bed fusion reactor by using the RELAP5-3D system code
}

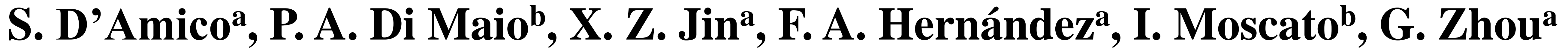

${ }^{a}$ Karlsruhe Institute of Technology (KIT), Eggenstein-Leopoldshafen, Germany

${ }^{b}$ Department of Engineering, University of Palermo, Palermo, Italy

\section{ABSTRACT}

In the frame of the activities promoted and encouraged by the EUROfusion Consortium aimed at developing the EU-DEMO fusion reactor, the research activity has been focused on the representative and safety relevant cooling loop of the Helium Cooled Pebble Bed (HCPB) Breeding Blanket (BB) Primary Heat Transfer System (PHTS), purposely selected by the safety team, in order to assess its thermal-hydraulic behaviour during normal operational conditions (ramp up/down and steady state) and to preliminarily investigate the consequences of an ex-vessel LOCA accidental scenario ensuing a double-ended guillotine (DEG) break in the hot leg. The research activity has been carried out following a theoretical-computational approach based on the finite volume method adopting the RELAP5-3D system code along with the ANSYS CFX computational fluid dynamic code, which were properly integrated to achieve a more detailed and realistic simulation of the EU-DEMO reactor thermal-hydraulics. Models, assumptions and outcomes of this preliminary study are herein presented and critically discussed.

The Finite Volume Model developed consists of four main sub-models:

- the flow domain sub-model, reproducing in a quasi-2D approximation the lay-out of the cooling circuit;

- the constitutive sub-models provided by the system code to describe the thermodynamic behaviour of the helium circulating inside the cooling system;

- the hydraulic sub-model intended to simulate the fluid flow along the cooling system.

- the thermal sub-model articulated in different sub-patterns aimed at realistically reproduce the heat transfer phenomena which take place along the cooling system.

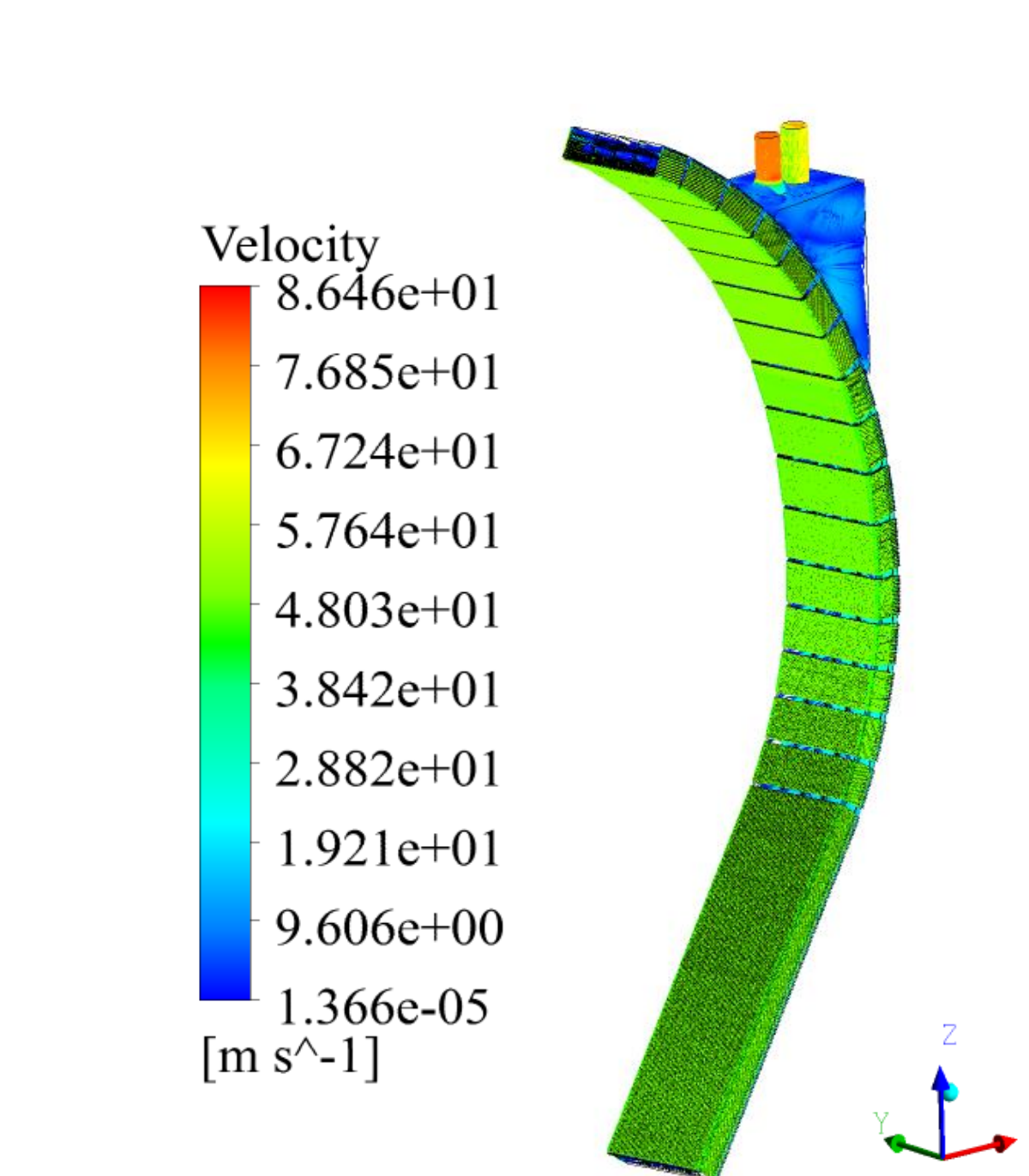

L.

\section{Finite Volume Model Set-up}

The thermal model has been assessed by performing an experimental benchmark with the data coming from a proper experience conducted on the HETRA facility.

A detailed CFD parametric analysis has been performed for the COB segment by using the porous media approach in order to assess the hydraulic characteristic function of its components giving the functional dependence of their total pressure drop on the corresponding mass flow rate:
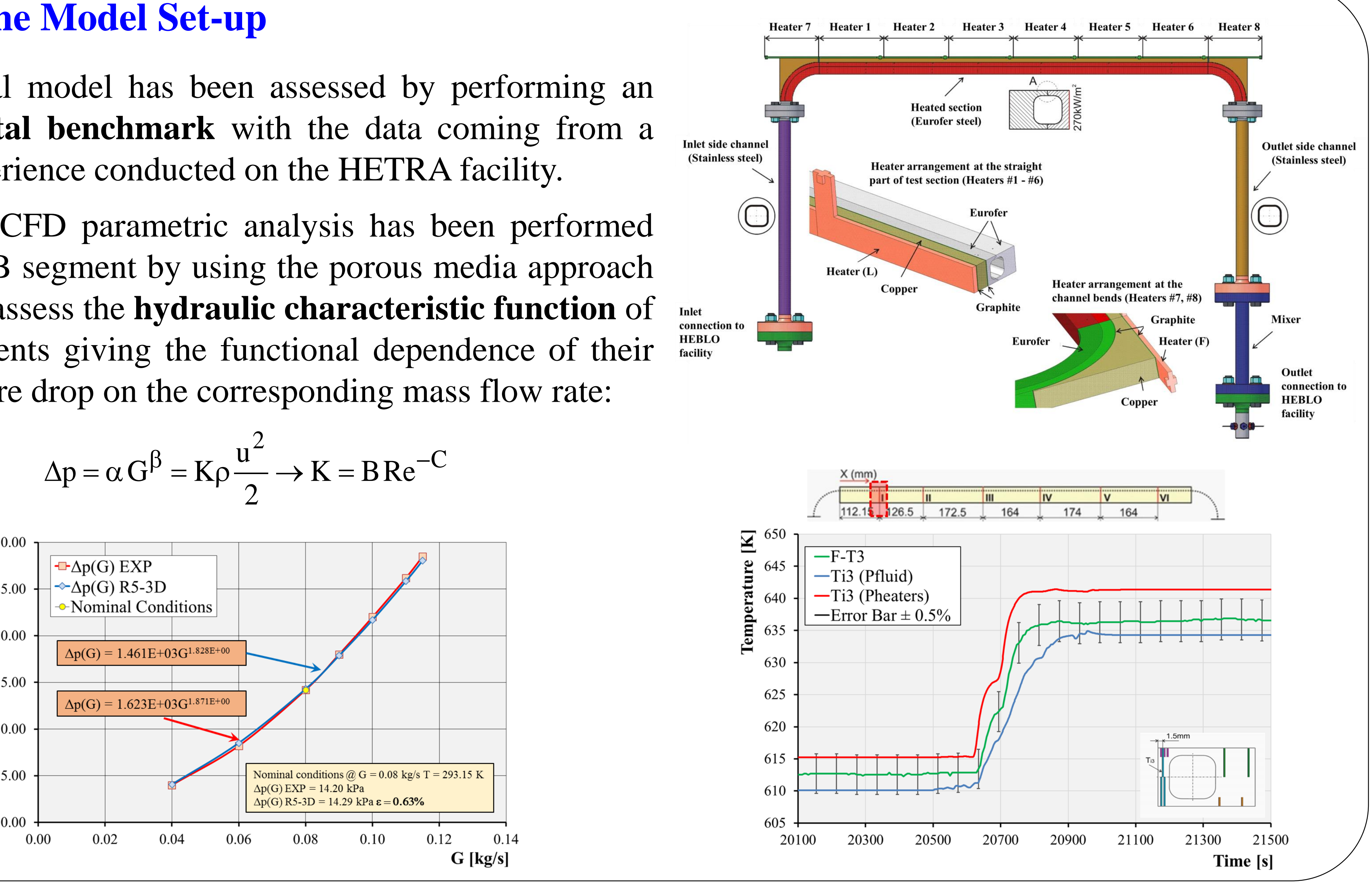

\section{RELAP5-3D Finite Volume Model}

It has been developed a realistic finite volume model for the safety relevant loop of the new BB-PHTS. Here, the noodalization scheme adopted for the cold, hot legs and that adopted to simulate each of the 10 segments.
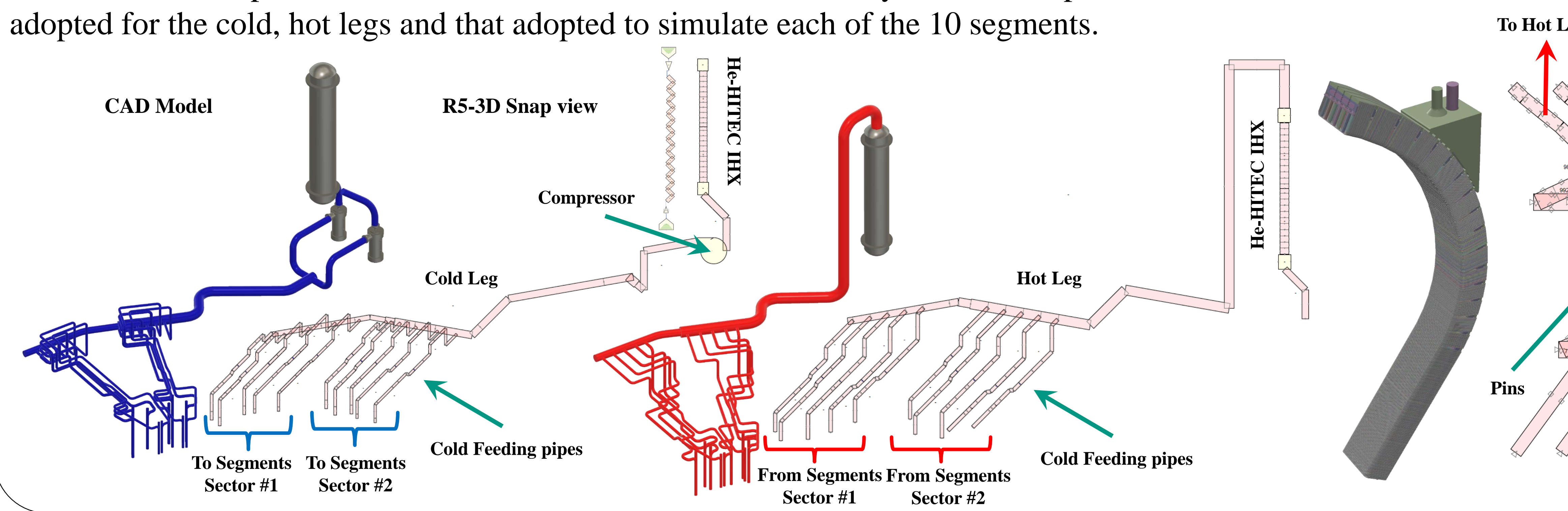

A parametric study has been performed analysing the normal operational conditions assessing the dynamic response of the system when it switches from pulse to dwell and vice versa. In particular, 4 exploratory cases involving a combination of different mass flow rates on both He and MS sides have been studied to evaluate the potential maximum variations that relevant quantities can experience for a given BoP control. Thereafter, a more suitable control has been analysed: He evolves with a viable rate of change of $12.5 \%$ per minute, $\mathrm{G}_{\mathrm{He} \_ \text {min }}=83.34 \mathrm{~kg} / \mathrm{s}$ during the dwell, whilst MS is controlled to maintain the required BB inlet temperature to $573.15 \mathrm{~K}$.
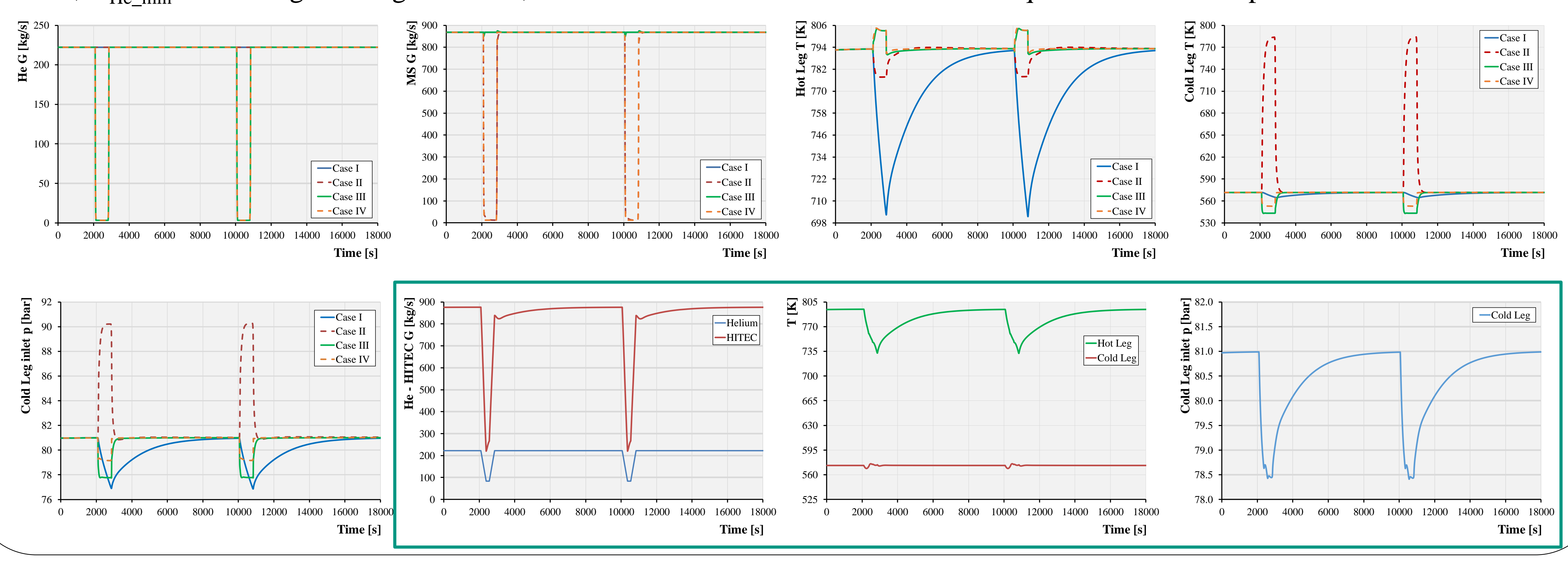

In order to qualify the nodalization, the pressure drops calculated by the code have been compared to the theoretical ones; the calculated total pressure drop is 2.58 while the theoretical value is 2.67 bar $(\varepsilon 3.41 \%)$

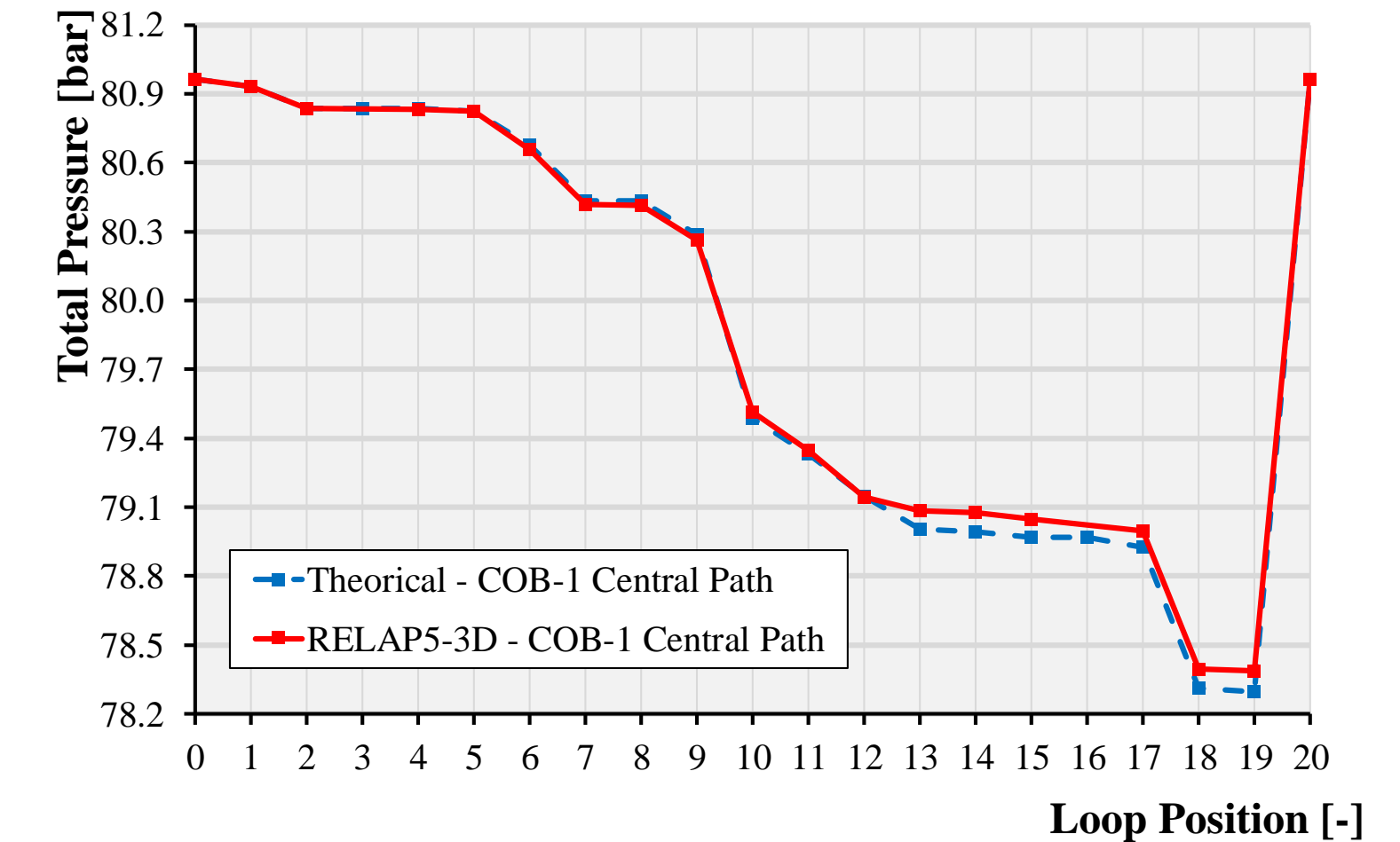

It has been investigated a DEG break occurring in hot leg where the cross section is $1.114 \mathrm{~m}^{2}$. The initial condition of the tokamak cooling rooms have been set to: $\mathrm{T}=333.15 \mathrm{~K}$ and $\mathrm{p}=95 \mathrm{kPa}$. The available volume is $1.18 \cdot 10^{5} \mathrm{~m}^{3}$

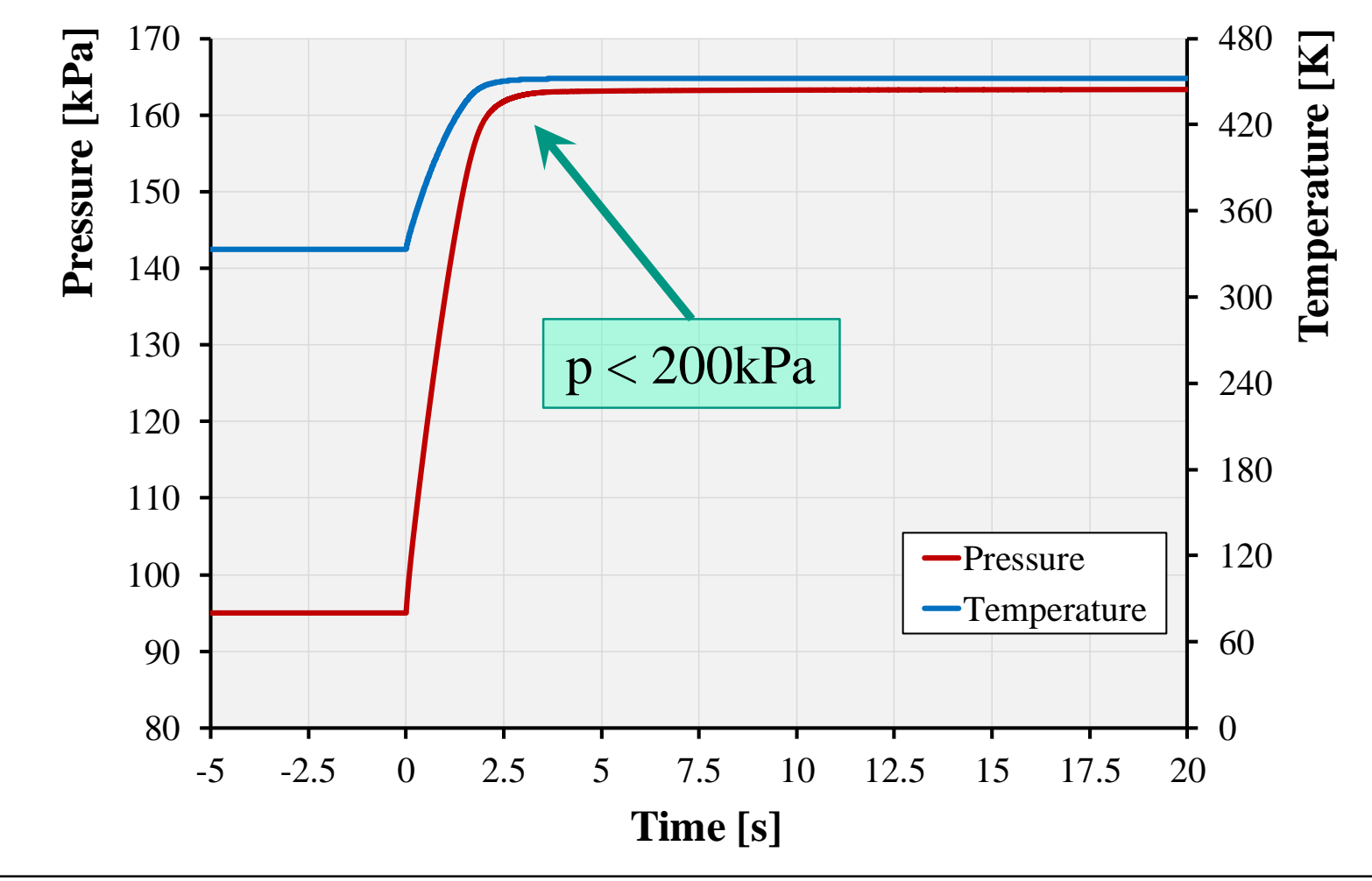

\section{CONCLUSION}

The results obtained simulating the normal operational scenario are in good agreement with the design data and the model seems suitable to investigate both operational and accidental transients suggesting the possibility to extend the thermal-hydraulic analyses to further the some of the scenarios following the PIEs already identified for the EU-DEMO HCPB reactor. 